\title{
Neuroelectromagnetic Correlates of Perceptual Closure Processes
}

\author{
Christine Grützner, ${ }^{1,2}$ Peter J. Uhlhaas, ${ }^{1,2}$ Erhan Genc, ${ }^{1}$ Axel Kohler, ${ }^{1,5}$ Wolf Singer, ${ }^{1,4}$ and Michael Wibral ${ }^{3}$ \\ ${ }^{1}$ Department of Neurophysiology, Max Planck Institute for Brain Research, ${ }^{2}$ Laboratory for Neurophysiology and Neuroimaging, Department of Psychiatry, \\ and ${ }^{3}$ Magnetoencephalography Unit, Brain Imaging Center, Johann Wolfgang Goethe University, 60528 Frankfurt am Main, Germany, ${ }^{4}$ Frankfurt Institute \\ for Advanced Studies, Johann Wolfgang Goethe University, 60438 Frankfurt Am Main, Germany, and ${ }^{5}$ Department of Psychiatric Neurophysiology, \\ University Hospital of Psychiatry, University of Bern, CH-3000 Bern 60, Switzerland
}

Perceptual closure refers to the coherent perception of an object under circumstances when the visual information is incomplete. Although the perceptual closure index observed in electroencephalography reflects that an object has been recognized, the full spatiotemporal dynamics of cortical source activity underlying perceptual closure processing remain unknown so far. To address this question, we recorded magnetoencephalographic activity in 15 subjects ( 11 females) during a visual closure task and performed beamforming over a sequence of successive short time windows to localize high-frequency gamma-band activity $(60-100 \mathrm{~Hz})$. Two-tone images of human faces (Mooney faces) were used to examine perceptual closure. Event-related fields exhibited a magnetic closure index between 250 and $325 \mathrm{~ms}$. Time-frequency analyses revealed sustained high-frequency gamma-band activity associated with the processing of Mooney stimuli; closure-related gamma-band activity was observed between 200 and $300 \mathrm{~ms}$ over occipitotemporal channels. Time-resolved source reconstruction revealed an early $(0-200 \mathrm{~ms})$ coactivation of caudal inferior temporal gyrus (cITG) and regions in posterior parietal cortex (PPC). At the time of perceptual closure $(200-400 \mathrm{~ms})$, the activation in cITG extended to the fusiform gyrus, if a face was perceived. Our data provide the first electrophysiological evidence that perceptual closure for Mooney faces starts with an interaction between areas related to processing of three-dimensional structure from shading cues (cITG) and areas associated with the activation of long-term memory templates (PPC). Later, at the moment of perceptual closure, inferior temporal cortex areas specialized for the perceived object are activated, i.e., the fusiform gyrus related to face processing for Mooney stimuli.

\section{Introduction}

An important ability of human vision is the recognition of objects even when only incomplete visual information is available. The term "perceptual closure" refers to the perception of an object that is not completely or immediately represented (Mooney, 1957; Snodgrass and Feenan, 1990). Changes in perceptual closure processes provide insights into cortical development (Uhlhaas et al., 2009) and impaired information processing in psychiatric disorders (Uhlhaas et al., 2006). Perceptual closure has often been studied using fragmented pictures of geometric figures and event-related potentials (ERPs) to outline the timing of neural processes underlying closure. Doniger et al. (2000) found a closure-related ERP component that was present over occipitotem-

\footnotetext{
Received Nov. 2, 2009; revised April 20, 2010; accepted April 27, 2010.

We thank Bill Phillips from the University of Stirling (Stirling, Scotland, UK), Christoph Bledowski and Benjamin Rahm from the Institute for Medical Psychology, Johann Wolfgang Goethe University (Frankfurt, Germany), Cerisa Stawowsky from the Department of Educational Psychology of the Johann Wolfgang Goethe University (Frankfurt, Germany), and Lucia Melloni and Eugenio Rodriguez from the Max Planck Institute for Brain Research (Frankfurt, Germany) for fruitful discussions and helpful comments on this manuscript. We also thank Ingrid Nieuwenhuis from the Donders Institute for Cognition, Brain, and Behaviour (Nijmegen, The Netherlands) for assistance with the implementation of the warped grids for the forward modeling.

This article is freely available online through the J Neurosci Open Choice option.

Correspondence should be addressed to Dr. Michael Wibral, Magnetoencephalography Unit, Brain Imaging Center, Johann Wolfgang Goethe University, Heinrich Hoffmann Strasse 10, 60528 Frankfurt am Main, Germany. E-mail: wibral@bic.uni-frankfurt.de.

D0I:10.1523/JNEUROSCI.5434-09.2010

Copyright $\odot 2010$ the authors $\quad 0270-6474 / 10 / 308342-11 \$ 15.00 / 0$
}

poral electrodes between 230 and $400 \mathrm{~ms}$. Source analyses localized the underlying neural generators in the lateral occipital complex (LOC) (Sehatpour et al., 2006), which has been related to object recognition (Malach et al., 1995; Grill-Spector et al., 1998).

It has been proposed that rhythmic synchronization of neural discharges in the gamma band $(>25 \mathrm{~Hz})$ plays an important role for the integration of visual features into a coherent object representation (Singer, 1999; Tallon-Baudry and Bertrand, 1999). Human electroencephalography (EEG) studies provided evidence that oscillatory activity in the gamma band is correlated with the perception of coherent objects (Keil et al., 1999; Rodriguez et al., 1999; Gruber and Müller, 2005; Martinovic et al., 2008). Most EEG gamma-band studies have focused on lower gamma-band frequencies $(<60 \mathrm{~Hz}$ ); however, recent magnetoencephalography (MEG) and intracranial EEG studies have shown that oscillations in the high-frequency gamma band $(>60 \mathrm{~Hz})$ are reliable markers of cortical activity during a variety of cognitive tasks (Crone et al., 2001; Kaiser et al., 2004; Brovelli et al., 2005; Lachaux et al., 2005; Hoogenboom et al., 2006; Vidal et al., 2006; Guggisberg et al., 2007; Siegel et al., 2007).

The present study examined the role of high-frequency gamma-band activity recorded with MEG during perceptual closure, using the Mooney faces task, a visual closure task consisting of two-tone face images (Mooney and Ferguson, 1951). Mooney faces provide three-dimensional (3D) structure from shading cues as the only low-level visual features, which are primarily 
A
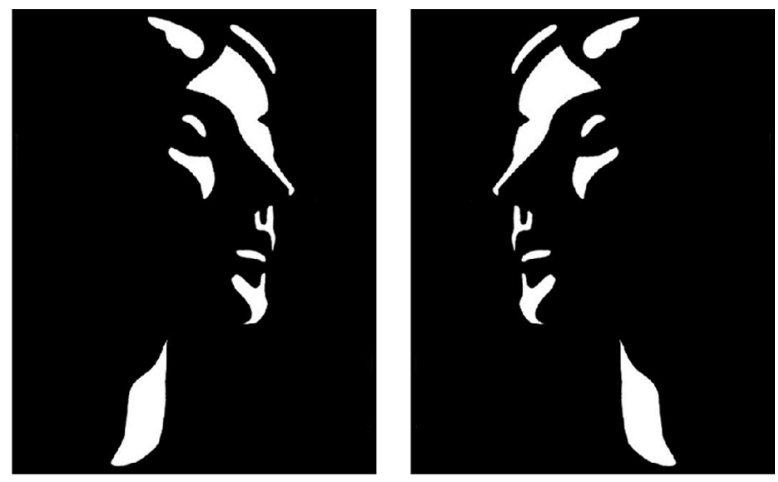

B

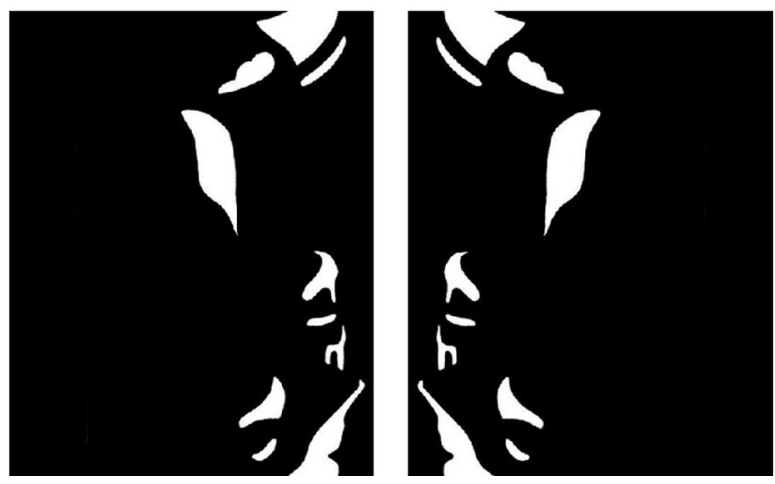

Figure 1. Examples of upright $(\boldsymbol{A})$ and inverted-scrambled $(\boldsymbol{B})$ Mooney face stimuli.

processed in the caudal inferior temporal gyrus (cITG) (Georgieva et al., 2008). However, the recognition of Mooney faces cannot be based on shading cues alone but is guided by top-down processes (Kemelmacher-Shlizerman et al., 2008), such as object familiarity (Dolan et al., 1997; Moore and Cavanagh, 1998) and memory processes (Dolan et al., 1997) represented in posterior parietal cortex (Wagner et al., 2005). In addition, functional magnetic resonance imaging (fMRI) studies have demonstrated that Mooney faces activate face-selective areas, such as the fusiform face area (FFA) (Kanwisher et al., 1998; Andrews and Schluppeck, 2004; McKeeff and Tong, 2007). Thus, we expected to find closure-related activation in these brain regions. The spatiotemporal dynamics at the level of cortical sources underlying perceptual closure has not been studied yet; thus, we reconstructed the underlying dynamic network of neural generators with timeresolved beamformer source analysis and additionally performed fMRI to further validate source localization.

\section{Materials and Methods \\ Subjects}

Fifteen healthy volunteers ( 11 females; mean \pm SD age, $25.4 \pm 5.6$ years) participated in the MEG experiment; 19 subjects (eight females; mean \pm SD age, $23.5 \pm 2.6$ years) participated in the fMRI part of our study. All subjects were right-handed as assessed by the Edinburgh Handedness Inventory (Oldfield, 1971) and had normal or corrected-to-normal visual acuity. All subjects gave written informed consent before the experiment. The study was approved by the local ethics committee (Johann Wolfgang Goethe University, Frankfurt, Germany).

\section{Stimuli}

The stimuli consisted of degraded pictures of faces in which all shades of gray were removed (Fig. 1) [Mooney Faces (Mooney and Ferguson, 1951)]. Mooney stimuli are typically recognized as faces only when presented in the upright orientation (Andrews and Schluppeck, 2004; George et al., 2005). We used a set of 160 different stimuli, consisting of the 40 original Mooney stimuli presented in the upright orientation, mirrored at the vertical axis and in corresponding versions mirrored at the horizontal axis. The inverted stimuli were scrambled by moving single contiguous white or black foreground patches across the black or white background areas, respectively. This scrambling ensured that no faces were perceived in the inverted stimulus condition. Importantly, upright and inverted-scrambled stimuli were matched with respect to low-level stimulus properties, such as luminance and spatial frequencies. Luminance of the white stimulus parts was $1140 \mathrm{~cd} / \mathrm{m}^{2}$ and of the black stimulus parts $30 \mathrm{~cd} / \mathrm{m}^{2}$. The stimuli were displayed in the center of a translucent screen at a viewing distance of $53 \mathrm{~cm}$ and subtended $19^{\circ}$ of visual angle. The background of the screen was set to gray $\left(145 \mathrm{~cd} / \mathrm{m}^{2}\right)$. A liquid crystal display projector located outside the magnetically shielded room of the MEG was used to project the stimuli onto the screen via two front-silvered mirrors. Stimulus presentation was controlled using the Presentation software package (version 11; Neurobehavioral Systems).

Task

Subjects were presented with a random sequence of upright and inverted-scrambled stimuli in each run. Each stimulus was presented for $200 \mathrm{~ms}$, with a random intertrial interval (ITI) between 3500 and 4500 $\mathrm{ms}$. Subjects were required to indicate whether they detected a face or not via button press after each stimulus. They were instructed to respond as quickly as possible and to fixate a central fixation cross during the ITI. Half of the subjects responded with their right index finger when they detected a face and with their left index finger when they did not. For the other half of subjects, response hands were reversed. Before data collection, subjects performed a practice block to become familiar with the task and the response buttons. All subjects completed six experimental runs, each of which was composed of 45 upright and 45 invertedscrambled stimuli.

In addition, we performed a rapid event-related fMRI experiment using the same stimuli and the same paradigm, except for the following changes. Each of the four fMRI runs contained 111 trials, consisting of a random number of face, non-face, and fixation trials. A two-back randomization procedure ensured that the order of presented stimuli was balanced within runs in each subject. Each trial was $4 \mathrm{~s}$ long, consisting of $200 \mathrm{~ms}$ stimulus duration and a fixed ITI of $3800 \mathrm{~ms}$, in which the fixation cross was presented. The stimulus was an upright Mooney face, an inverted-scrambled Mooney face, or just the fixation cross. Thus, the duration of the interval between successive Mooney stimuli presentations was at least the length of the fixed ITI (3800 ms), which was followed by a random number of fixation trials between one and four volumes; including these fixation trials provided the jitter between face and non-face presentations necessary for rapid event-related fMRI. Each run began and ended with an $8 \mathrm{~s}$ fixation period. Subjects performed four fMRI runs within one scanning session.

\section{Data acquisition}

MEG data were recorded continuously using a 275-channel whole-head system (Omega 2005; VSM MedTech Ltd.) at a rate of $600 \mathrm{~Hz}$ in a synthetic third-order axial gradiometer configuration (Data Acquisition Software version 5.4.0; VSM MedTech Ltd.). The data were filtered with fourth-order Butterworth filters with $0.5 \mathrm{~Hz}$ high pass and $150 \mathrm{~Hz}$ low pass. Behavioral responses were recorded using a fiber-optic response pad (Lumitouch, Photon Control) on the stimulus personal computer and fed through to the MEG acquisition system as an additional channel. Before and after each run, the subject's head position relative to the gradiometer array was measured using coils placed at the subject's nasion and $1 \mathrm{~cm}$ anterior to the tragus of the left and right ear. Runs with a head movement exceeding $5 \mathrm{~mm}$ were discarded. Cushions were used to stabilize subjects' heads inside the MEG helmet.

Structural and functional magnetic resonance images were obtained with a $3 \mathrm{~T}$ Siemens Allegra scanner using the standard circularly polarized birdcage head coil. Functional images were acquired using a $\mathrm{T} 2{ }^{*}$ weighted echo planar imaging sequence sensitive to blood oxygenation level-dependent (BOLD) contrast [repetition time (TR), $1000 \mathrm{~ms}$; echo time (TE), $30 \mathrm{~ms}$; flip angle, $60^{\circ}$ ]. Eighteen axial slices covering occipital cortex, parietal cortex, frontal lobes, and the posterior part of the temporal lobe were collected [field of view (FOV), $220 \mathrm{~mm}$; in-plane resolu- 
tion, $3.3 \times 3.3 \mathrm{~mm}$; slice thickness, $5 \mathrm{~mm}$; gap thickness, $0.5 \mathrm{~mm}$ ]. These slices did not cover the anterior inferotemporal cortex (aIT) and the inferior frontal cortex, limiting our later analysis of correspondence between MEG and fMRI source locations in these regions. Each functional run was preceded by eight volumes that were discarded to allow for magnetization to reach a stable state. A $3 \mathrm{D}$ magnetization-prepared rapid-acquisition gradient echo sequence (160 slices; voxel size, $1 \times 1 \times$ $1 \mathrm{~mm}$; FOV, $256 \mathrm{~mm}$; TR, $2300 \mathrm{~ms}$; TE, $3.93 \mathrm{~ms}$ ) was used to obtain high-resolution anatomical images. These anatomical images were used to coregister and display functional data and to create individual head models for MEG source reconstruction (see below). During the structural scan, vitamin E pills were placed $1 \mathrm{~cm}$ anterior to the tragus of the right and left ear to allow for coregistration of the MEG and MRI data.

\section{Data analysis}

$M E G$ data analysis. MEG data were analyzed using the open source Matlab toolbox Fieldtrip (version 2008-12-08; http://www.ru.nl/fcdonders/ fieldtrip/). For data preprocessing, data epochs were defined from the continuously recorded MEG signals from - 1000 to $1000 \mathrm{~ms}$ with respect to the onset of the visual stimulus. Data epochs were sorted according to the two experimental conditions: the face condition, containing trials with upright stimuli, and the non-face condition, containing trials with inverted-scrambled stimuli. Only data epochs with correct responses were considered for all additional analyses, i.e., hits for the face condition and correct rejections for the non-face condition. Data epochs contaminated by eye blinks, muscle activity, or jump artifacts in the sensors were discarded using automatic artifact detection and rejection routines from the Fieldtrip toolbox. Data epochs were baseline corrected by subtracting the mean amplitude during an epoch ranging from -500 to $-100 \mathrm{~ms}$ before stimulus onset.

We performed three analyses. First, we analyzed event-related magnetic fields (ERFs) to identify an MEG-ERF analog of the perceptual closure index $(\mathrm{Ncl})$ observed in previous EEG-ERP studies (Doniger et al., 2000). Second, we analyzed task-related changes in spectral power associated with perceptual closure at the sensor level. This analysis served to determine the frequencies of interest for later source analysis and to demonstrate closure-related effects at the sensor level. Third, and most important to the aim of our study, the electrical generators of these spectral components of interest were localized in the brain using a frequency domain beamformer (Gross et al., 2001) as implemented in Fieldtrip (version 2008-12-08; function beamformer_dics.m with real valued filter coefficients) on successive short time windows. We used the frequency domain beamformer to estimate local source power (Gross et al., 2001, their Formula 5), not source coherence estimates. Therefore, we constrained the analysis to real valued spatial filters, to reflect instantaneous linear mixing from sources to sensors. This latter constraint is dropped when dynamic imaging of coherent sources as implemented in the above Fieldtrip function is used to image source power that is coherent to an external reference (e.g., the electromyogram signal) and coupling with a delay is expected.

Event-related fields. ERFs were obtained by averaging the segmented trials in the time range between -500 and $500 \mathrm{~ms}$ separately for each subject in each condition. For each subject, the ERF to non-faces was subtracted from the ERF to faces to assess the amplitude and the latency of the event-related activity associated with closure processes. We tested the obtained ERFs for effects of activation versus baseline and differences between conditions in the interval from 200 to $350 \mathrm{~ms}$ using randomization testing with a cluster-based threshold correction method (Maris and Oostenveld, 2007; for an application, see Wibral et al., 2009) to correct for multiple comparisons. A cluster $p$ value below $5 \%$ (two-tailed testing) was considered significant. Source analysis for event-related fields was performed with minimum norm estimation (Hämäläinen and Ilmoniemi, 1994) for the time interval between 250 and 300 ms. Normalized lead fields and the same head model as in the beamforming analysis (see below) were used. The regularization parameter was $5 \%$. We performed a randomization test with $p<0.01$ (uncorrected) for statistical testing of minimum norm estimates.

Analysis of event-related spectral power changes at the sensor level. Timefrequency representations (TFRs) were computed by means of Morlet wavelets with a width of five cycles per wavelet at center frequencies between 25 and $150 \mathrm{~Hz}$, in $1 \mathrm{~Hz}$ steps. Time-frequency transformations were computed in a time window of $-500 \mathrm{~ms}$ before stimulus to 500 $\mathrm{ms}$ after stimulus. We tested the obtained time-frequency transformations for effects of activation $(50-350 \mathrm{~ms})$ versus baseline $(-350$ to $-50 \mathrm{~ms}$ ) and for differences between conditions in the interval between 50 and 350 ms using the same randomization testing and cluster-based threshold correction method (Maris and Oostenveld, 2007) that was also used for the ERF analysis. Again, a cluster $p$ value below 5\% (two-tailed testing) was considered significant. In addition, we performed a correction for multiple comparisons using the false discovery rate (FDR) $(q<$ 0.05) (Genovese et al., 2002). A frequency window for the beamformer source analysis of the generators of oscillatory sensor-level signals was chosen by inspecting the time-frequency transformation for significant task-related effects that lasted for at least $200 \mathrm{~ms}$.

Reconstruction of the sources of oscillatory sensor-level components: beamforming parameters. We used a frequency domain beamformer (Gross et al., 2001) at the frequencies of interest that had been identified at the sensor level. Beamformers are spatial filters that project sensor activity to specified source locations in the brain using a linearly weighted sum of the sensor signals. Source power from a given location is reconstructed with unit gain while interference from all other source locations is suppressed as much as possible. Because beamformer source analysis exhibits a bias for erroneously high power values for the center of the head, source power values have to be normalized appropriately. This is typically achieved by comparing the source power of interest from the task interval to that obtained from a corresponding baseline interval (dual-state beam forming) (Huang et al., 2004 and references therein).

We performed the frequency domain beamformer analyses for a sequence of overlapping, short intervals (duration, $200 \mathrm{~ms}$; onsets at every $50 \mathrm{~ms}$ from 0 to $450 \mathrm{~ms}$ ). We decided to perform time-resolved beamforming to examine power differences between conditions because sensor-level analysis of condition-specific spectral power changes had revealed transient effects with a duration on the order of 100-200 ms. The center frequency for the frequency domain beamformer was $80 \mathrm{~Hz}$. The cross-spectral density matrices were computed using the multi-taper method (Percival and Walden, 1993) with four Slepian tapers (Slepian, 1978), which lead to a spectral smoothing of $20 \mathrm{~Hz}$. To compensate for the short duration of the data windows, we used a regularization of $\lambda=$ $5 \%$ (Brookes et al., 2008). The baseline interval for comparison in dualstate beamforming was chosen from -350 to $-150 \mathrm{~ms}$ before stimulus onset.

Time-resolved dual-state beamforming and statistical procedures at source level. To investigate differences in source power between faces and non-faces, we computed the frequency domain beamformer filters for combined data epochs ("common filters") consisting of activation (duration, $200 \mathrm{~ms}$; onsets at every $50 \mathrm{~ms}$ from 0 to $450 \mathrm{~ms}$ ) and baseline data $(-350$ to $-150 \mathrm{~ms})$ for each analysis interval. Our analysis thus covered a total time interval from 0 to $650 \mathrm{~ms}$. Computing common filters for task and baseline data later enabled a statistical test of the hypothesis that source power is changed by a stimulus. In contrast, when computing separate sets of filters for task and baseline, the hypothesis to be tested is that either source power changes or beamformer filters differ between task and baseline because of a changing number of active sources between task and baseline (Nieuwenhuis et al., 2008, their Methods). We then projected the sensor data through these common filters for each trial, separately for task and baseline. Next, we computed a within-subject randomization test statistic (pseudo- $t$ ) based on these single-trial data. We thus obtained a test statistic for activation versus baseline effects for each grid point, each subject, and each condition. The values of this test statistic were subjected to a second-level randomization test at the multisubject level to obtain groupwise effects of differences between conditions; a $p$ value $<0.01$ was considered significant.

Source location grid. The set of potential source locations, i.e., the source grid, was constructed as follows. First, we overlaid a regular threedimensional dipole grid with an isotropic spacing of $10 \mathrm{~mm}$ on the $\mathrm{T} 1$ template of the SPM2 toolbox (http://www.fil.ion.ucl.ac.uk/spm), provided by the Montreal Neurological Institute (MNI) (Montreal, Quebec, Canada; http://www.bic.mni.mcgill.ca/brainweb). Next, we transformed 
each individual subject's anatomical MRI onto this template using the linear transformation from SPM2 and recorded each subject's individual transformation matrix. Then we warped the regular dipole grid with the inverse of each individual transformation matrix. Thus, we obtained specific dipole grids for each subject, enabling us to perform beamformer source analysis with individual head models. The corresponding forward solution (lead field) for each subject was computed using its individual dipole grid and a realistic single-shell volume conductor model (Hämäläinen and Sarvas, 1987, 1989) with an implementation described by Nolte et al. (2003). Importantly, each location of the dipole grid in MNI space had a unique corresponding grid location in each subject. This enabled multisubject statistics over corresponding anatomical locations in each subject.

fMRI data analysis. fMRI data were analyzed using the Brain Voyager QX software package (version 1.10.3; Brain Innovation). The first four volumes of each event-related run were discarded to preclude T1 saturation effects. Preprocessing of the functional data included motion correction, linear trend removal to remove baseline drifts, temporal high-pass filtering at $0.01 \mathrm{~Hz}$ to remove slow fluctuations in the BOLD signal time course, and slice scan-time correction. Spatial smoothing was performed with a Gaussian filter with a $6 \mathrm{~mm}$ kernel for the group analysis. For each subject, the functional and structural 3D datasets were normalized to Talairach space (Talairach and Tournoux, 1988). The BOLD signal time courses for the different experimental conditions were estimated using a deconvolution analysis (Burock et al., 1998; Glover, 1999). Twenty predictors were defined for each condition to cover the temporal extent of a typical hemodynamic response. For statistical analysis, a multisubject fixed-effects general linear model was computed on the three peak time points of the BOLD signal. Linear contrasts of faces minus non-faces were computed to identify closure-related activity. Coordinates for activated clusters were tabulated and converted to MNI space (Collins, 1994) for comparison with MEG source locations by means of the WFU PickAtlas (Maldjian et al., 2003) and the Jülich histological atlas (Eickhoff et al., 2007) provided by FSL (http://www.fmrib.ox.ac.uk/fsl).

\section{Results}

\section{Performance}

We analyzed the percentage of correct responses as well as reaction times in correct trials for the face and the non-face conditions. Paired $t$ tests were computed to test the behavioral data for significance. The performance accuracy did not differ significantly between the face and the non-face conditions (faces correct: mean $\pm \mathrm{SD}, 81.13 \pm 5.84 \%$; non-faces correct: $82.47 \pm$ $\left.14.32 \% ; t_{(14)}=-0.28 ; p=0.78\right)$. Reaction times were significantly longer in the non-face condition (faces RT: mean $\pm \mathrm{SD}$, $723.81 \pm 131.6 \mathrm{~ms}$; non-faces RT: $801.07 \pm 144.19 \mathrm{~ms} ; t_{(14)}=$ $-3.39 ; p=0.004)$.

\section{Event-related fields}

To assess closure-related activity, we computed the difference between ERFs in the face and in the non-face conditions between 200 and $350 \mathrm{~ms}$ after stimulus onset. Differences were most pronounced between 250 and $300 \mathrm{~ms}$ (Fig. 2C, bottom row). These differences were observed bilaterally at sensors over lateral occipital and temporal cortex. Potential sources of these differences were localized in temporal (middle and superior temporal gyrus), parietal (precuneus and inferior parietal lobule), and occipital brain areas (Fig. $2 E$, bottom row).

\section{Event-related spectral power changes on the sensor level}

Time-frequency spectral power was analyzed in a broad frequency band (25-150 Hz), including both the low-frequency $(25-60 \mathrm{~Hz})$ and the high-frequency $(60-150 \mathrm{~Hz})$ gamma bands. For the presentation of upright Mooney stimuli, we found increases in event-related spectral power in a cluster of parietooccipital sensors (Fig. 3A, top). These effects were present in both the lower and higher gamma band. Time- and frequencyresolved analysis revealed transient broadband effects at latencies at $\sim 50$ and $250 \mathrm{~ms}$ after stimulus onset, most likely related to stimulus-locked activity in response to onset and offset (at 200 $\mathrm{ms}$ ) of the visual stimulus. In addition, we found sustained significant increases in event-related spectral power in a more confined frequency band from 60 to $100 \mathrm{~Hz}$ in the interval from $\sim 100$ to $300 \mathrm{~ms}$ (Fig. 3A, bottom, left). Significant decreases in event-related spectral power were found at a cluster of frontal and central sensors (Fig. 3A, top). This cluster was dominated by power changes in the lower gamma-frequency range at latencies starting at $\sim 150 \mathrm{~ms}$ and lasting until the end of the analysis interval (Fig. 3A, bottom, right). Event-related changes in spectral power for inverted Mooney stimuli (Fig. $3 B$ ) were similar in time and frequency to those found for upright Mooney stimuli.

For faces compared with non-faces, we found a significantly stronger decrease in event-related power at frontocentral sensors at latencies from 200 to $350 \mathrm{~ms}$ (Fig. 3C, top, top row). This effect was dominated by power changes in the lower gamma-frequency range. In other words, both conditions induced task-related power decreases at these sensors, latencies, and frequencies; the decrease was significantly stronger in the face condition. This effect was the only effect reaching significance at the level of a cluster-based threshold. When using FDR $(q<0.05)$ to correct for multiple comparisons, a more diverse picture emerged (Fig. $3 C$, middle and bottom rows). Although we found a complex pattern of significant changes over sensors, latencies, and frequencies, its main features can be described as follows. Between 200 and $250 \mathrm{~ms}$, i.e., in the interval in which the perceptual closure index was found in EEG studies, there was significantly higher event-related spectral power in the lower and higher gamma-frequency band at temporal-occipital sensors for the face compared with the non-face condition. In the time intervals from 100 to $150 \mathrm{~ms}$ and from $250 \mathrm{~ms}$ to the end of the analysis interval, we found a significantly stronger power decrease for the face compared with the non-face condition at frontal and temporal sensors.

\section{Beamformer source analysis}

Source power was estimated for a sequence of short, overlapping intervals in the high-frequency gamma band $(60-100 \mathrm{~Hz})$. Figure 4 shows the differences in reconstructed source power between faces and non-faces, and Table 1 lists the corresponding anatomical location as coordinates in MNI space. In the first time intervals (onset times from 0 to $150 \mathrm{~ms}$ ), source power was predominantly stronger in the face condition compared with the non-face condition (red clusters, numbers 1-7, 10, and 11). Enhanced source power for faces was observed in parietal regions, more specifically in the right superior parietal lobule/precuneus and the left angular/supramarginal gyrus. Furthermore, occipitotemporal cortex showed enhanced activation for the face condition in the left lateral occipital complex (LOC)/cITG. Finally, regions in the temporal lobe, including the right superior temporal gyrus and left and right aIT, were more strongly activated for faces compared with non-faces. In the time interval between 150 and $350 \mathrm{~ms}$, there were two clusters in the right frontal cortexone in the middle frontal gyrus (MiFG) and the other one in inferior frontal gyrus (IFG)-with stronger activation for the non-face compared with the face condition (blue clusters, numbers 8 and 9).

During the time interval in which the perceptual closure index was found in EEG studies (200-400 ms, "closure interval") (Doniger et al., 2000), we observed enhanced activity for faces 
A
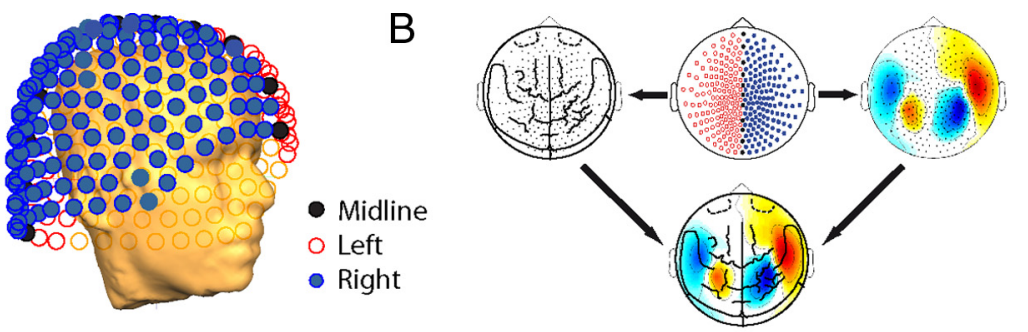

C

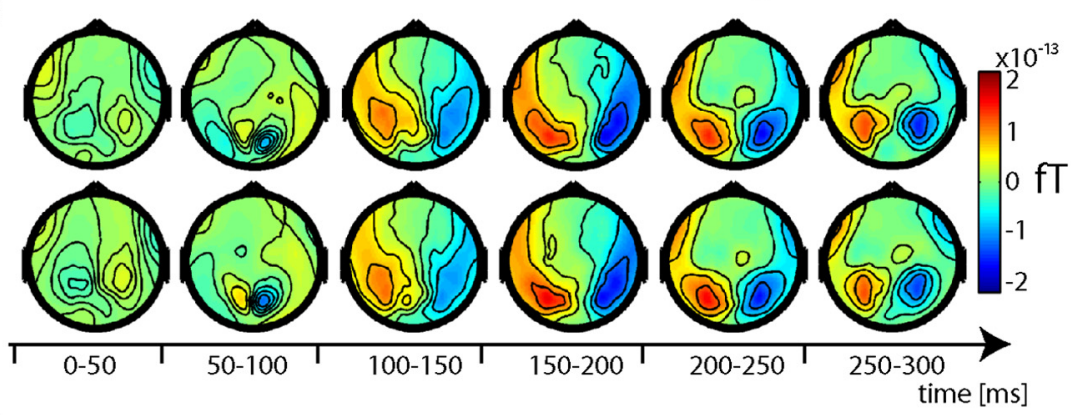

D

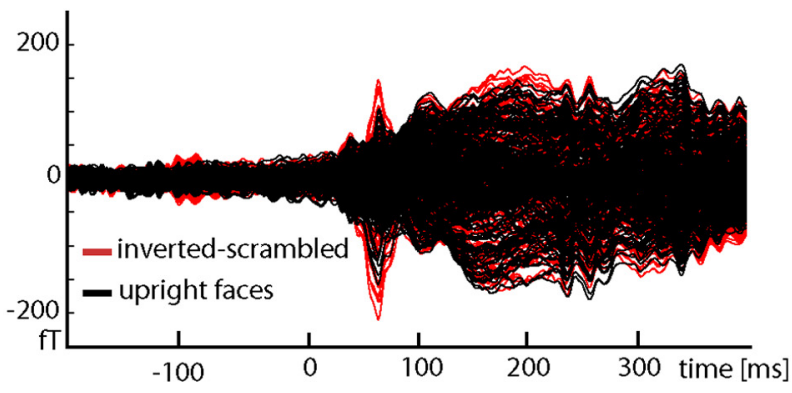

$\mathrm{E}$

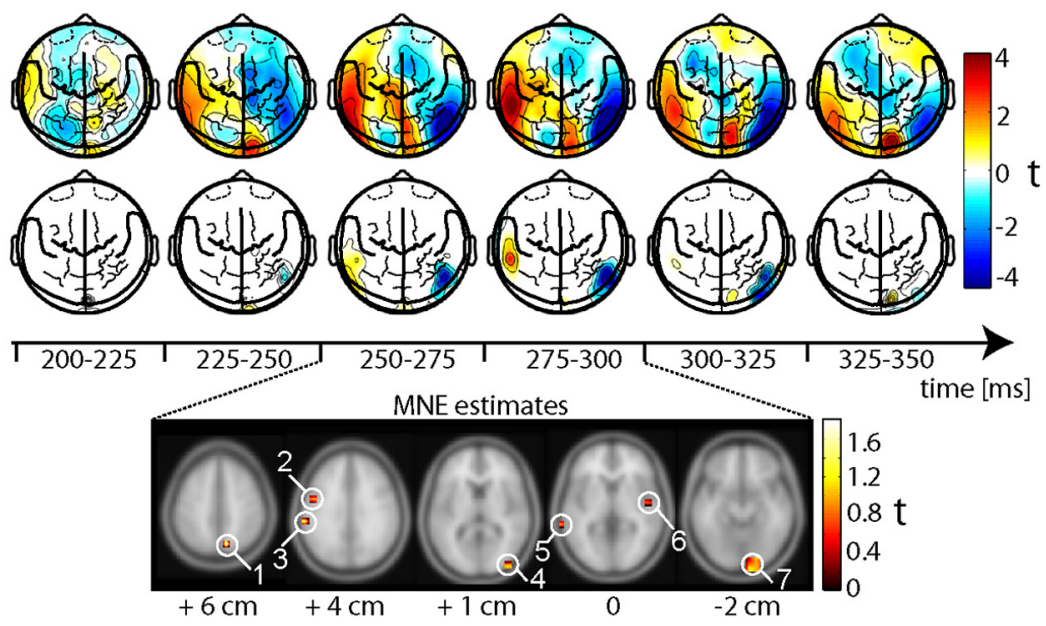

$\mathrm{F}$

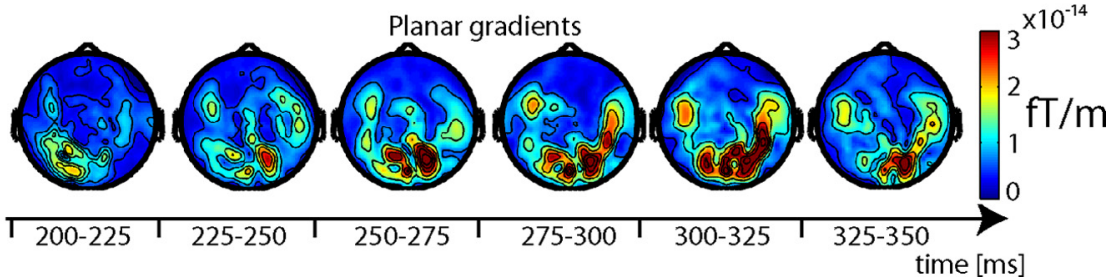

Figure 2. A, $3 \mathrm{D}$ arrangement of the $275 \mathrm{MEG}$ sensors around the subject's head. B, Plotting conventions for $2 \mathrm{D}$ sensor-level topographies. Top row, Middle, All sensors are plotted inside a schematic top view of the head (colors refer to the 3D plot in $A$ ). Coregistration between a reconstructed 2D topography of the major anatomical landmarks of the brain (top row, left) (Kaiser et al., 2002) and the sensor positions (top row, right). Bottom row, Interpolated sensor-level topographies coregistered with the major anatomical landmarks via sensor positions. This display type is used for the remainder of this study. C, Raw, baseline-corrected event-related field topographies for the face (top row) and the non-face (bottom row) conditions. D, Corresponding event-related field traces for all sensors. Red, Non-face condition; black, face condition. $\boldsymbol{E}$, Significant differences between event-related magnetic fields in the face minus the non-face condition. Top row, Sensor-level topographies of $t$ values over time. Middle row Sensor-level topographies masked by a statistical significance mask ( $p<0.05$ corrected). Bottom row, Statistical differences compared with non-faces in the left cITG; this cluster also extended to the fusiform gyrus (FFA). Furthermore, there was enhanced activity for faces in the left IFG and also in the left aIT. FFA and left IFG were activated more strongly for faces specifically during the closure interval, whereas the aIT showed enhanced activation for faces during almost all time windows. As in the preceding time interval, the right IFG showed stronger activation for nonfaces compared with faces.

During later time intervals (onset times from 250 to $450 \mathrm{~ms}$ ), the activation pattern shifted from primarily enhanced activation for faces to primarily enhanced activation for non-faces. The main clusters showing enhanced source power for non-faces were located in the right frontal cortex, more specifically in the MiFG, in the IFG, and also in the premotor cortex. Additional clusters with stronger activation for non-faces were found in primary visual areas between 300-500 and 350$550 \mathrm{~ms}$ and in the anterior cingulate gyrus between $400-600$ and $450-650 \mathrm{~ms}$.

\section{fMRI data}

To obtain an independent measure of the brain regions that were differentially activated for the face and non-face condition, we repeated the same experiment with fMRI. Overall, there was a very close correspondence between the MEG source locations and regions showing differential activation in fMRI (Fig. 5, Table 1). In the fMRI data, however, we found bilateral activation for most areas (except for the MiFG and the premotor cortex), whereas the MEG source reconstructions showed mostly clusters lateralized to only one hemisphere. This might be attributable to differences in analysis techniques because MEG beamformer filters tend to misplace or suppress correlated sources (Huang et al., 2004, their Fig. 2; Hillebrand et al., 2005), which in combination with thresholding might mask certain sources. The cluster in the premotor cortex was located in the right hemisphere in

$\leftarrow$

between the face and non-face conditions for minimum norm source amplitude estimates ( $p<0.01$, uncorrected) for signals averaged from 250 to $300 \mathrm{~ms}$. White circles indicate significant clusters. Cluster 1, Precuneus (Talairach coordinates: $10,-66,49)$; cluster 2 , precentral gyrus $(-48,3,28)$; cluster 3 , inferior parietal lobule $(-59,-29,33)$; cluster 4 , middle occipital gyrus $(-42,-87,14)$; cluster 5 , middle temporal gyrus $(-67,-39,2)$; cluster 6 , superior temporal gyrus (54, $-10,-1)$; cluster 7 , lingual gyrus $(16,-94,-5)$. F, Absolute value of the planar gradient of raw field differences between face and non-face conditions. 

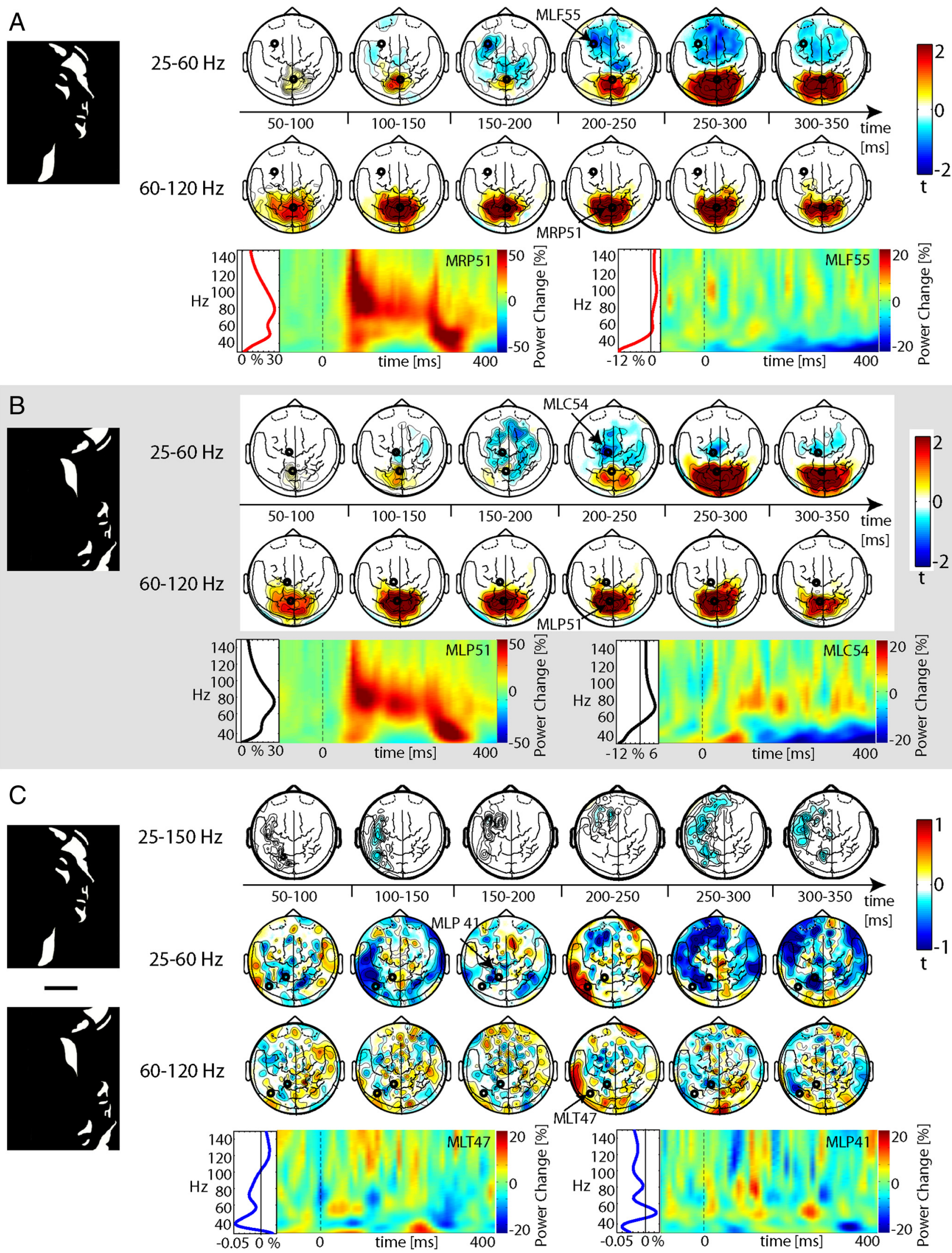

Figure 3. Statistical analysis of power changes in response to upright Mooney faces $(\boldsymbol{A})$, inverted Mooney faces $(\boldsymbol{B})$, and for the difference between upright and inverted stimuli $(\boldsymbol{C}$. All data represent grand averages or statistical analysis across all subjects. The top part of each panel shows the temporal evolution of the topography of the statistical analysis; bottom, each panel contains power spectra and TFRs for specific sensors. Topographies, $A, B$, Significant differences between the respective Mooney stimulus condition and the $0.5 \mathrm{~s}$ prestimulus baseline, separately for the (Figure legend continues.) 
the MEG data but in the left hemisphere in the fMRI data. We did not cover the IFG and the temporal pole in the fMRI experiment, meaning that it was a priori not possible to find fMRI activations in these regions. Therefore, we cannot draw any conclusions about a correspondence between MEG and $\mathrm{fMRI}$ activations regarding these areas (clusters 4, 5, and 7).

\section{Discussion}

We examined the spatiotemporal dynamics underlying perceptual closure using MEG recordings, time-resolved beamforming, and cross-validation of source localization by fMRI. Our data provide evidence that distributed neural generators in occipitotemporal, parietal, and frontal areas are involved in highfrequency gamma-band activity $(60-100 \mathrm{~Hz})$ in the processing of Mooney images.

Upright and inverted Mooney stimuli elicited sustained spectral amplitude increases in the high-frequency gamma band (60$100 \mathrm{~Hz}$ ) that were present for the entire stimulus duration, suggesting that the network relevant to our task operated prominently in the high gamma-frequency range. EEG studies have shown that coherent object representations are associated with increases in gamma-band activity (for review, see Tallon-Baudry and Bertrand, 1999; Keil et al., 2001), but effects in these studies were mainly in the lower gamma band $(<60 \mathrm{~Hz})$. Compatible with our findings, however, recent MEG and intracranial EEG studies demonstrated gamma-band responses between 60 and $200 \mathrm{~Hz}$ during a variety of cognitive tasks (Crone et al., 2001; Kaiser et al., 2004; Brovelli et al., 2005; Lachaux et al., 2005; Hoogenboom et al., 2006; Siegel et al., 2007; Guggisberg et al., 2007), suggesting that oscillations in the high gamma-frequency range $(>60 \mathrm{~Hz})$ might be important to cortical function in general.

The comparison between upright and inverted Mooney stimuli at the sensor level revealed differences rapidly changing over frequencies, sensors, and time. A pronounced enhancement of gamma-band activity was observed for faces in a time window between 200 and $300 \mathrm{~ms}$ at occipitotemporal sensors. This finding agrees with a previous EEG study on neural synchronization during the perception of Mooney faces, showing that spectral power in the gamma band differentiated between the perception and the nonperception of a face $\sim 230 \mathrm{~ms}$ (Rodriguez et al., 1999).

\footnotetext{
$\leftarrow$

(Figure legend continued.) lower $(25-60 \mathrm{~Hz})$ and the higher $(60-120 \mathrm{~Hz})$ gamma band. The effect is masked by the spatiotemporal pattern of the two significant clusters that resulted from the nonparametric cluster-based test. Red denotes the positive cluster (higher activation during stimulus presentation compared with baseline), and blue denotes the negative cluster (less activation during stimulus presentation compared with baseline). C, Significant differences between the face and the non-face condition. Top row, Power differences masked by the significant cluster that resulted from the nonparametric cluster-based statistical testing. Blue denotes higher activation for the non-face condition compared with the face condition. The middle and the bottom row of $C$ show the same topography as the top row but masked by the significance map derived from FDR $(q<0.05)$ statistical testing, tested together but plotted separately for the lower (middle row) and higher (bottom row) gamma band. Power spectra and TFRs are shown for the sensors with the most pronounced positive and negative effects. These sensors are marked by black circles in the topographic plots. In $\boldsymbol{A}$ and $\boldsymbol{B}$, the effect is expressed as percentage change of the power in the poststimulus window compared with baseline. In C, channel MLT47 represents the channel with the strongest positive effect of the difference between the face and the non-face condition (face $>$ non-face), whereas channel MLP41 is the channel with the strongest negative effect of the difference between the face and the non-face condition (face $<$ non-face). The power spectra are shown as a function of frequency $(25-150 \mathrm{~Hz})$, averaged for the time window between 0 and $400 \mathrm{~ms}$. The TFRs are plotted for the frequency range between 25 and $150 \mathrm{~Hz}$ and the time interval between -100 to $400 \mathrm{~ms}$. The dashed lines indicate stimulus onset. Also see supplemental material (available at www.jneurosci.org) for an analysis using multitapers.
}

The time range and the topography of closure-related oscillatory activity in the present study also corresponds with the timing of the perceptual closure index found in EEG studies (Doniger et al., 2000; Sehatpour et al., 2006) and to the ERFs in the present study. The complex pattern of differences between upright and inverted Mooney stimuli suggests that the network underlying perceptual closure comprises a number of different sources that are difficult to interpret at the sensor level.

The analysis of the sources underlying perceptual closure revealed a network of distributed sources in occipitotemporal, parietal, and frontal regions, which were differentially activated during specific time intervals. In early time ranges (0-200 ms), activity was mostly enhanced for faces, mainly in parietal and occipitotemporal areas. The activation in occipitotemporal areas was located in cITG and in the aIT. Georgieva et al. (2008) demonstrated that cITG is activated when a $3 \mathrm{D}$ object is reconstructed from shading cues. Our finding of stronger activation in cITG for Mooney face stimuli supports our initial hypothesis that the extraction of 3D shape cues provided by shading (KemelmacherShlizerman et al., 2008) is indeed essential for the perception of Mooney faces.

Furthermore, we found enhanced source power for faces in the aIT, which has been related to face processing (Kriegeskorte et al., 2007; Tsao et al., 2008). The aIT cluster in our data was located more posterior compared with the activation observed by Kriegeskorte and colleagues; this discrepancy could be attributable to inaccuracies in MEG beamforming or fMRI localization near the petruous bone. Kriegeskorte and colleagues proposed that FFA detects faces and engages aIT for identification. In the current data, however, the aIT cluster was activated throughout almost all time intervals and even before activation of the FFA, suggesting that it either plays a more general role in face processing or that information about individual faces is used very early and throughout the processing of Mooney faces to aid the detection of faces.

Enhanced activation for faces in parietal areas was located in the superior parietal lobe/precuneus in the right hemisphere and in the angular/supramarginal gyrus in the left hemisphere. Activation in posterior parietal cortex has been implicated in memory processes, especially in matching sensory input to long-term memory (Wagner et al., 2005). The medial parietal cortex (precuneus) has been related to memory-related imagery; it has been proposed that the precuneus reflects the "mind's eye" during reconstruction of object (or face) representations from fragmentary evidence (Fletcher et al., 1995; Dolan et al., 1997). Our findings support the view that memory-related processes play a critical role for the perception of Mooney faces. We suggest that, during the early stages of Mooney face processing, shapeprocessing areas in cITG may interact with parietal areas related to retrieval of face templates from memory.

During the closure interval (200-400 ms), the activation in cITG extended medially to the fusiform gyrus if a face was perceived. The fusiform gyrus is well known to be involved in face processing (Kanwisher et al., 1997). Thus, at the time of closure, the interaction between shape-processing areas (cITG) and category-specific areas (fusiform gyrus) seems to be required for object identification. An additional cluster in the left IFG showed enhanced activation for faces specifically in the closure interval, suggesting that the IFG is involved in perceptual closure processes. This interpretation is in line with a recent study that investigated perceptual recognition processes with fMRI and found that activation of the IFG was related to the moment of recognition (Ploran et al., 2007). 


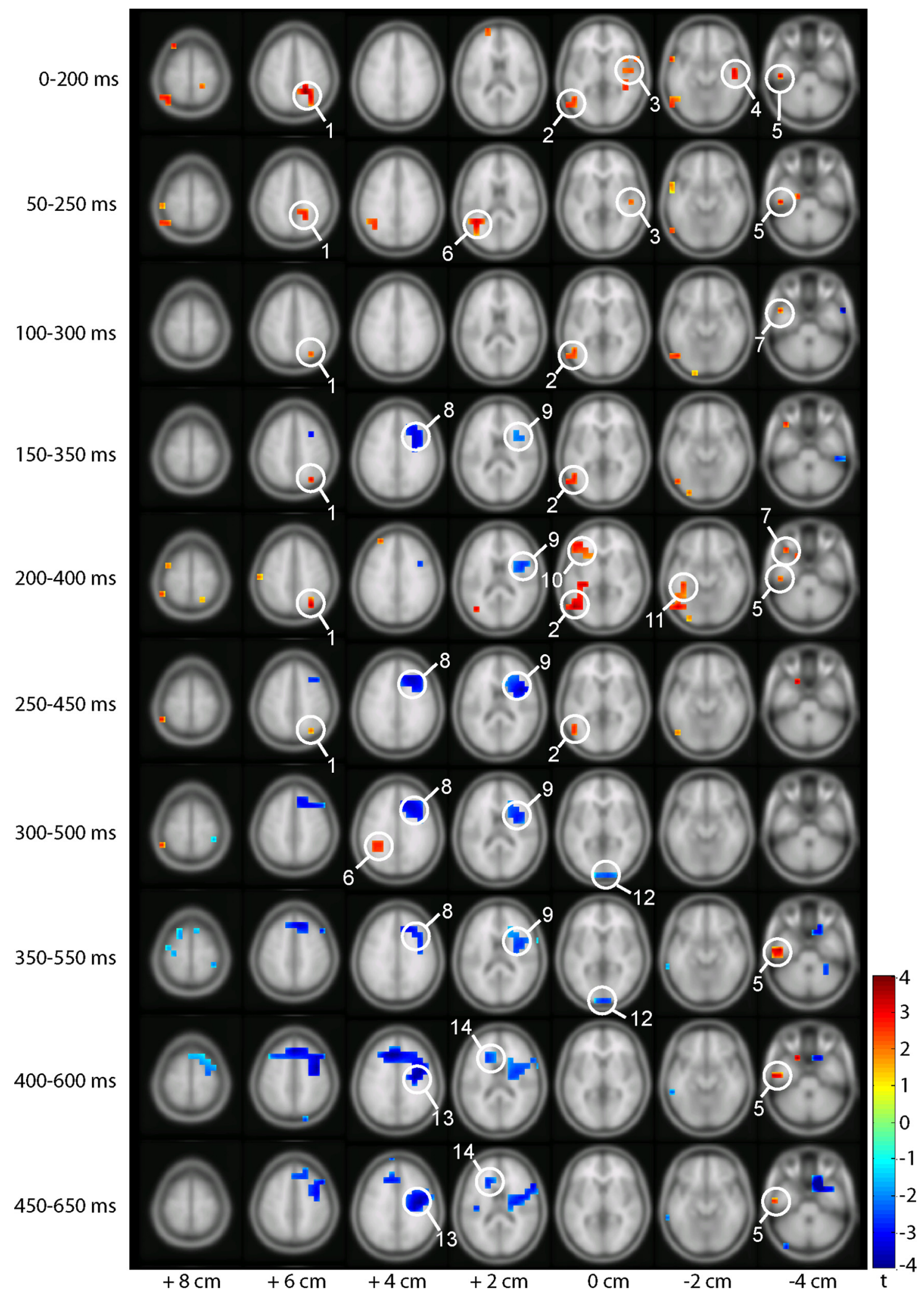

Figure 4. Differences in source power at $80 \mathrm{~Hz}$ between the face and the non-face condition ( $t$ values masked by $p<0.01$, uncorrected). Time intervals are plotted from top to bottom, and consecutive brain slices are plotted from left to right for each time interval (slice distance, $20 \mathrm{~mm}$ ) in neurological convention. Each cluster is marked on one slice per time interval, with the circles representing the center of each cluster (1-14). Red clusters represent stronger source power for faces; blue clusters represent stronger source power for non-faces ( $t$ values masked by $p<0.01$, not corrected). 1, Superior parietal lobe; 2, cITG; 3, superior temporal gyrus; 4, right alT; 5, left alT; 6, angular/supramarginal gyrus; 7, orbitofrontal cortex; 8, MiFG; 9, right IFG; 10, left IFG; 11, FFA; 12, primary visual cortex; 13, premotor cortex; 14, cingulate gyrus. The coordinates for all clusters are given in Table 1. 
Table 1. Anatomic locations and coordinates for clusters with differential gamma source power between upright and inverted-scrambled Mooney faces

\begin{tabular}{|c|c|c|c|c|c|c|c|}
\hline \multirow[b]{2}{*}{ Cluster } & \multirow[b]{2}{*}{ Anatomic location } & \multicolumn{3}{|l|}{ MEG } & \multicolumn{3}{|l|}{ fMRI } \\
\hline & & $x$ & $y$ & $z$ & $x$ & $y$ & $Z$ \\
\hline 1 & R superior parietal lobule/precuneus & 27 & -60 & 51 & 33 & -62 & 52 \\
\hline 2 & L caudal ITG/LOC & -50 & -69 & -4 & -49 & -69 & -10 \\
\hline 3 & R superior temporal gyrus & 50 & -13 & -5 & 53 & -14 & -4 \\
\hline 4 & R anterior inferotemporal cortex & 54 & -16 & -20 & & & \\
\hline 5 & L anterior inferotemporal cortex & -52 & -16 & -30 & & & \\
\hline 6 & L angular/supramarginal gyrus & -40 & -53 & 33 & -40 & -57 & 34 \\
\hline 7 & L orbitofrontal cortex & -36 & 21 & -31 & & & \\
\hline 8 & $\mathrm{R}$ middle frontal gyrus & 37 & 22 & 37 & 34 & 30 & 42 \\
\hline 9 & R inferior frontal gyrus & 40 & 19 & 17 & & & \\
\hline 10 & L inferior frontal gyrus & -44 & 36 & 0 & & & \\
\hline 11 & L fusiform gyrus & -40 & -46 & -10 & -41 & -49 & -15 \\
\hline 12 & R primary visual cortex & 2 & -100 & -2 & -6 & -98 & 4 \\
\hline 13 & R premotor cortex & 44 & 2 & 39 & -39 & -24 & 48 \\
\hline 14 & L cingulate gyrus & -13 & 29 & 24 & -9 & 27 & 27 \\
\hline
\end{tabular}

L, Left; R, right. $x, y, z$ coordinates are in MNI space. Anatomic locations are approximate.

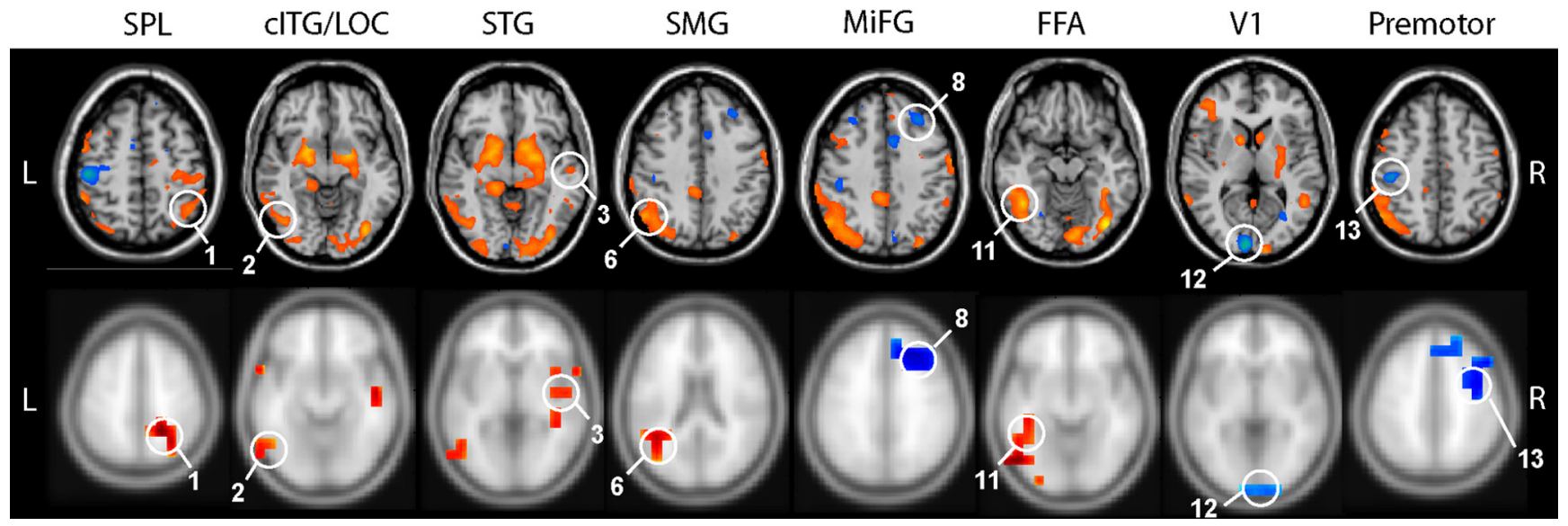

Figure 5. Differential fMRI activation maps for faces minus non-faces, overlaid on the structural image of one subject at a statistical threshold of $q<0.05$ (FDR, corrected) and corresponding contrasts for MEG beamforming. Orange voxels showed greater activation for faces than non-faces, whereas blue voxels showed greater activation for non-faces than faces. Clusters corresponding to fMRI (top row) and MEG (bottom row) source locations are marked with white circles; the numbers refer to the clusters in Table 1 and in Figure 4 . The clusters 4 (right alT), 5 (left alT), 7 (orbitofrontal cortex), 9 (right IFG), and 10 (left IFG) are missing because these areas were not covered during the functional MRI scans. SPL, Superior parietal lobule; STG, superior temporal gyrus; SMG, supramarginal gyrus; V1, primary visual cortex; Premotor, premotor cortex; L, left; $R$, right.

Interestingly, in time windows succeeding the closure interval (window centers at $350 \mathrm{~ms}$ and later), activation was predominantly enhanced for inverted stimuli, mainly in frontal regions located in the IFG, MiFG, and premotor cortex. It has been shown that the frontal lobe is activated during cognitively demanding tasks (Duncan and Owen, 2000). This stronger frontal activation for inverted stimuli may represent increased cognitive demand, assuming that subjects try to detect a face in each picture. Additionally, we observed stronger activity in the premotor cortex for inverted compared with upright stimuli in this later time interval. This finding could be related to the fact that subjects had significantly longer reaction times in the inverted condition. Primary visual cortex also showed stronger activation for inverted compared with upright stimuli in the later task phases, which could reflect the reaccess of early visual memory traces to use low-level cues for the reconstruction of a face. The stronger activation could also reflect increased attention.

The localization of closure-related brain areas in the present study is consistent with numerous studies on object recognition that demonstrated a widespread distribution of sources in inferior temporal, parietal, and frontal regions (Dolan et al., 1997; Lachaux et al., 2005; Gruber et al., 2008; Martinovic et al., 2008). Our findings suggest that high-frequency gamma oscillations as recorded by MEG allow a reliable reconstruction of perceptual closure processes with high spatiotemporal resolution. However, source locations obtained from MEG beamforming alone should not be taken to represent the true locations with zero error. This is because beamforming is limited by the ill-posed inverse problem (Grave de Peralta-Menendez and Gonzalez-Andino, 1998) and will also fail in the presence of sources that are highly correlated (Van Veen et al., 1997), leading to missing or mislocalized sources. This limitation applies even when statistical tests are performed because source locations may be systematically biased. Here, we tried to alleviate this problem by independently estimating sources of differential activity from fMRI data using the same visual closure task. This approach was motivated by the correlation between high-frequency oscillatory activity and BOLD fMRI signals (Logothetis et al., 2001; Brookes et al., 2005; Niessing et al., 2005). For those source locations that were covered by fMRI, we found a close match between source locations obtained from beamforming and fMRI (Table 1). Interestingly, sources did not only match in location but also with respect to the sign of the differences between the two conditions. Therefore, we are confident that the reported MEG source locations are close to the actual neural generators for the sources covered by fMRI. It is unclear at present why some bilateral fMRI sources were only 
unilaterally reported in MEG. Possible reasons include statistical thresholding and extinction or mislocalization of source power attributable to intersource correlation. Hence, statements on lateralization of the processes of interest are not warranted by the current dataset.

\section{Conclusion}

The present study examined the mechanisms of visual closure processing at an unprecedented level of spatial and temporal detail combining fMRI and a time-resolved MEG beamforming approach. The reconstruction of the neural generators of highfrequency activity revealed closure-related activity in a distributed network of sources in occipitotemporal, parietal, and frontal brain areas. In early task phases $(0-200 \mathrm{~ms})$, there was a coactivation of cITG-a region related to processing of 3D structure from shading cues-and regions in posterior parietal cortex that have been related to activation of long-term memory templates. At the time of perceptual closure (200-400 ms), the activation in cITG extended to the fusiform gyrus if a face was perceived, suggesting that the perceptual closure index is related to activation in inferior temporal cortex areas specialized for the perceived object. Thus, the present study suggests that perceptual closure is based on an early interaction of object recognition and memoryrelated areas.

\section{References}

Andrews TJ, Schluppeck D (2004) Neural responses to Mooney images reveal a modular representation of faces in human visual cortex. Neuroimage 21:91-98.

Brookes MJ, Gibson AM, Hall SD, Furlong PL, Barnes GR, Hillebrand A, Singh KD, Holliday IE, Francis ST, Morris PG (2005) GLMbeamformer method demonstrates stationary field, alpha ERD and gamma ERS co-localisation with fMRI BOLD response in visual cortex. Neuroimage 26:302-308.

Brookes MJ, Vrba J, Robinson SE, Stevenson CM, Peters AM, Barnes GR, Hillebrand A, Morris PG (2008) Optimising experimental design for MEG beamformer imaging. Neuroimage 39:1788-1802.

Brovelli A, Lachaux JP, Kahane P, Boussaoud D (2005) High gamma frequency oscillatory activity dissociates attention from intention in the human premotor cortex. Neuroimage 28:154-164.

Burock MA, Buckner RL, Woldorff MG, Rosen BR, Dale AM (1998) Randomized event-related experimental designs allow for extremely rapid presentation rates using functional MRI. Neuroreport 9:3735-3739.

Collins DL (1994) 3D model-based segmentation of individual brain structures from magnetic resonance imaging data. PhD thesis, McGill University.

Crone NE, Boatman D, Gordon B, Hao L (2001) Induced electrocorticographic gamma activity during auditory perception. Brazier Awardwinning article, 2001. Clin Neurophysiol 112:565-582.

Grave de Peralta-Menendez R, Gonzalez-Andino SL (1998) A critical analysis of linear inverse solutions to the neuroelectromagnetic inverse problem. IEEE Trans Biomed Eng 45:440-448.

Dolan RJ, Fink GR, Rolls E, Booth M, Holmes A, Frackowiak RS, Friston KJ (1997) How the brain learns to see objects and faces in an impoverished context. Nature 389:596-599.

Doniger GM, Foxe JJ, Murray MM, Higgins BA, Snodgrass JG, Schroeder CE, Javitt DC (2000) Activation timecourse of ventral visual stream objectrecognition areas: high density electrical mapping of perceptual closure processes. J Cogn Neurosci 12:615-621.

Duncan J, Owen AM (2000) Common regions of the human frontal lobe recruited by diverse cognitive demands. Trends Neurosci 23:475-483.

Eickhoff SB, Paus T, Caspers S, Grosbras MH, Evans AC, Zilles K, Amunts K (2007) Assignment of functional activations to probabilistic cytoarchitectonic areas revisited. Neuroimage 36:511-521.

Fletcher PC, Frith CD, Baker SC, Shallice T, Frackowiak RS, Dolan RJ (1995) The mind's eye: precuneus activation in memory-related imagery. Neuroimage 2:195-200.

Genovese CR, Lazar NA, Nichols T (2002) Thresholding of statistical maps in functional neuroimaging using the false discovery rate. Neuroimage $15: 870-878$.

George N, Jemel B, Fiori N, Chaby L, Renault B (2005) Electrophysiological correlates of facial decision: insights from upright and upside-down Mooney-face perception. Brain Res Cogn Brain Res 24:663-673.

Georgieva SS, Todd JT, Peeters R, Orban GA (2008) The extraction of 3D shape from texture and shading in the human brain. Cereb Cortex $18: 2416-2438$

Glover GH (1999) Deconvolution of impulse response in event-related BOLD fMRI. Neuroimage 9:416-429.

Grill-Spector K, Kushnir T, Edelman S, Itzchak Y, Malach R (1998) Cueinvariant activation in object-related areas of the human occipital lobe. Neuron 21:191-202.

Gross J, Kujala J, Hamalainen M, Timmermann L, Schnitzler A, Salmelin R (2001) Dynamic imaging of coherent sources: studying neural interactions in the human brain. Proc Natl Acad Sci U S A 98:694-699.

Gruber T, Müller MM (2005) Oscillatory brain activity dissociates between associative stimulus content in a repetition priming task in the human EEG. Cereb Cortex 15:109-116.

Gruber T, Maess B, Trujillo-Barreto NJ, Müller MM (2008) Sources of synchronized induced gamma-band responses during a simple object recognition task: a replication study in human MEG. Brain Res 1196:74-84.

Guggisberg AG, Dalal SS, Findlay AM, Nagarajan SS (2007) High-frequency oscillations in distributed neural networks reveal the dynamics of human decision making. Front Hum Neurosci 1:14.

Hämäläinen MS, Ilmoniemi RJ (1994) Interpreting magnetic fields of the brain: minimum norm estimates. Med Biol Eng Comput 32:35-42.

Hämäläinen MS, Sarvas J (1987) Feasibility of the homogeneous head model in the interpretation of neuromagnetic fields. Phys Med Biol 32:91-97.

Hämäläinen MS, Sarvas J (1989) Realistic conductivity geometry model of the human head for interpretation of neuromagnetic data. IEEE Trans Biomed Eng 36:165-171.

Hillebrand A, Singh KD, Holliday IE, Furlong PL, Barnes GR (2005) A new approach to neuroimaging with magnetoencephalography. Hum Brain Mapp 25:199-211.

Hoogenboom N, Schoffelen JM, Oostenveld R, Parkes LM, Fries P (2006) Localizing human visual gamma-band activity in frequency, time and space. Neuroimage 29:764-773.

Huang MX, Shih JJ, Lee RR, Harrington DL, Thoma RJ, Weisend MP, Hanlon F, Paulson KM, Li T, Martin K, Millers GA, Canive JM (2004) Commonalities and differences among vectorized beamformers in electromagnetic source imaging. Brain Topogr 16:139-158.

Kaiser J, Lutzenberger W, Ackermann H, Birbaumer N (2002) Dynamics of gamma-band activity induced by auditory pattern changes in humans. Cereb Cortex 12:212-221.

Kaiser J, Bühler M, Lutzenberger W (2004) Magnetoencephalographic gamma-band responses to illusory triangles in humans. Neuroimage 23:551-560.

Kanwisher N, McDermott J, Chun MM (1997) The fusiform face area: a module in human extrastriate cortex specialized for face perception. J Neurosci 17:4302-4311.

Kanwisher N, Tong F, Nakayama K (1998) The effect of face inversion on the human fusiform face area. Cognition 68:B1-B11.

Keil A, Müller MM, Ray WJ, Gruber T, Elbert T (1999) Human gamma band activity and perception of a gestalt. J Neurosci 19:7152-7161.

Keil A, Gruber T, Müller MM (2001) Functional correlates of macroscopic high-frequency brain activity in the human visual system. Neurosci Biobehav Rev 25:527-534.

Kemelmacher-Shlizerman I, Basri R, Nadler B (2008) 3D shape reconstruction of Mooney faces. In: IEEE Conference on Computer Vision Pattern Recognition, Anchorage, AK, June.

Kriegeskorte N, Formisano E, Sorger B, Goebel R (2007) Individual faces elicit distinct response patterns in human anterior temporal cortex. Proc Natl Acad Sci U S A 104:20600-20605.

Lachaux JP, George N, Tallon-Baudry C, Martinerie J, Hugueville L, Minotti L, Kahane P, Renault B (2005) The many faces of the gamma band response to complex visual stimuli. Neuroimage 25:491-501.

Logothetis NK, Pauls J, Augath M, Trinath T, Oeltermann A (2001) Neurophysiological investigation of the basis of the fMRI signal. Nature 412:150-157.

Malach R, Reppas JB, Benson RR, Kwong KK, Jiang H, Kennedy WA, Ledden 
PJ, Brady TJ, Rosen BR, Tootell RB (1995) Object-related activity revealed by functional magnetic resonance imaging in human occipital cortex. Proc Natl Acad Sci U S A 92:8135-8139.

Maldjian JA, Laurienti PJ, Kraft RA, Burdette JH (2003) An automated method for neuroanatomic and cytoarchitectonic atlas-based interrogation of fMRI data sets. Neuroimage 19:1233-1239.

Maris E, Oostenveld R (2007) Nonparametric statistical testing of EEG- and MEG-data. J Neurosci Methods 164:177-190.

Martinovic J, Gruber T, Hantsch A, Müller MM (2008) Induced gammaband activity is related to the time point of object identification. Brain Res 1198:93-106.

McKeeff TJ, Tong F (2007) The timing of perceptual decisions for ambiguous face stimuli in the human ventral visual cortex. Cereb Cortex 17:669-678.

Mooney CM (1957) Age in the development of closure ability in children. Can J Psychol 11:219-226.

Mooney CM, Ferguson GA (1951) A new closure test. Can J Psychol 5:129-133.

Moore C, Cavanagh P (1998) Recovery of 3D volume from 2-tone images of novel objects. Cognition 67:45-71.

Niessing J, Ebisch B, Schmidt KE, Niessing M, Singer W, Galuske RA (2005) Hemodynamic signals correlate tightly with synchronized gamma oscillations. Science 309:948-951.

Nieuwenhuis IL, Takashima A, Oostenveld R, Fernández G, Jensen O (2008) Visual areas become less engaged in associative recall following memory stabilization. Neuroimage 40:1319-1327.

Nolte G (2003) The magnetic lead field theorem in the quasi-static approximation and its use for magnetoencephalography forward calculation in realistic volume conductors. Phys Med Biol 48:3637-3652.

Oldfield RC (1971) The assessment and analysis of handedness: the Edinburgh inventory. Neuropsychologia 9:97-113.

Percival DB, Walden AT (1993) Spectral analysis for physical applications: multitaper and conventional univariate techniques. Cambridge, UK: Cambridge UP.

Ploran EJ, Nelson SM, Velanova K, Donaldson DI, Petersen SE, Wheeler ME (2007) Evidence accumulation and the moment of recognition: dissociating perceptual recognition processes using fMRI. J Neurosci 27:11912-11924.

Rodriguez E, George N, Lachaux JP, Martinerie J, Renault B, Varela FJ (1999) Perception's shadow: long-distance synchronization of human brain activity. Nature 397:430-433.
Sehatpour P, Molholm S, Javitt DC, Foxe JJ (2006) Spatiotemporal dynamics of human object recognition processing: an integrated high-density electrical mapping and functional imaging study of "closure" processes. Neuroimage 29:605-618.

Siegel M, Donner TH, Oostenveld R, Fries P, Engel AK (2007) Highfrequency activity in human visual cortex is modulated by visual motion strength. Cereb Cortex 17:732-741.

Singer W (1999) Neuronal synchrony: a versatile code for the definition of relations? Neuron 24:49-65, 111-125.

Slepian D (1978) Prolate spheroidal wave functions, Fourier analysis, and uncertainty. V. The discrete case. Bell System Tech J 57:1371-1430.

Snodgrass JG, Feenan K (1990) Priming effects in picture fragment completion: support for the perceptual closure hypothesis. J Exp Psychol Gen 119:276-296

Talairach J, Tournoux P (1988) Co-planar stereotaxic atlas of the human brain. New York: Thieme Medical Publishers.

Tallon-Baudry C, Bertrand O (1999) Oscillatory gamma activity in humans and its role in object representation. Trends Cogn Sci 3:151-162.

Tsao DY, Moeller S, Freiwald WA (2008) Comparing face patch systems in macaques and humans. Proc Natl Acad Sci U S A 105:19514-19519.

Uhlhaas PJ, Linden DE, Singer W, Haenschel C, Lindner M, Maurer K, Rodriguez E (2006) Dysfunctional long-range coordination of neural activity during Gestalt perception in schizophrenia. J Neurosci 26:8168-8175.

Uhlhaas PJ, Roux F, Singer W, Haenschel C, Sireteanu R, Rodriguez E (2009) The development of neural synchrony reflects late maturation and restructuring of functional networks in humans. Proc Natl Acad Sci U S A 106:9866-9871.

Van Veen BD, van Drongelen W, Yuchtman M, Suzuki A (1997) Localization of brain electrical activity via linearly constrained minimum variance spatial filtering. IEEE Trans Biomed Eng 44:867-880.

Vidal JR, Chaumon M, O’Regan JK, Tallon-Baudry C (2006) Visual grouping and the focusing of attention induce gamma-band oscillations at different frequencies in human magnetoencephalogram signals. J Cogn Neurosci 18:1850-1862.

Wagner AD, Shannon BJ, Kahn I, Buckner RL (2005) Parietal lobe contributions to episodic memory retrieval. Trends Cogn Sci 9:445-453.

Wibral M, Bledowski C, Kohler A, Singer W, Muckli L (2009) The timing of feedback to early visual cortex in the perception of long-range apparent motion. Cereb Cortex 19:1567-1582. 